\title{
Compact-Open and Point Wise Convergence Topologies
}

\author{
Mohammed Nokhas Murad Kaki \\ Department of Mathematics, College of Science, University of Sulaimani, Sulaimani, Iraq \\ Email: Muradkakaee@yahoo.com
}

How to cite this paper: Kaki, M.N.M. (2018) Compact-Open and Point Wise Convergence Topologies. Open Access Library Journal, 5: e4775.

https://doi.org/10.4236/oalib.1104775

Received: July 13, 2018

Accepted: August 11, 2018

Published: August 14, 2018

Copyright $\odot 2018$ by author and Open Access Library Inc.

This work is licensed under the Creative Commons Attribution International License (CC BY 4.0).

http://creativecommons.org/licenses/by/4.0/

\begin{abstract}
In this paper, we have investigated and introduced some new definitions of transitivity on the set of all continuous maps, denoted by $C(X, Y)$, called the point-wise convergence transitive, the compact-open transitive and point wise convergence topological transitive sets. Relationship between these new definitions is studied. Finally, we have introduced a number of very important topological concepts and shown that every compact-open convergence transitive map implies point wise transitive maps but the converse not necessarily true.
\end{abstract}

\section{Subject Areas}

Mathematical Analysis

\section{Keywords}

Compact-Open Topology, Transitive Set, Chaotic Sets, Point Wise Convergence Mixing

\section{Introduction}

Let $(X, \tau)$ and $(Y, \sigma)$ be two topological spaces and $C(X, Y)$ be the set of all continuous maps from $X$ into $Y$. Consider all possible sets of maps of the form

$$
[K, U]=\{f \in C(X, Y): f(K) \subset U\},
$$

where $K$ is a compact set in $X$ and $U$ an open set in $Y$. The topology $\tau_{3}$ generated by these sets $[K, U]$ as a subbase is called the compact-open topology on $C(X, Y)$. Note that any open set in $\tau_{3}$ is called co-open set and $\left(C(X, Y), \tau_{3}\right)$ is called co-topological space. The compliment of co-open set is called co-closed 
set. We have introduced some new definitions of transitivity on $C(X, Y)$, called the point-wise convergence transitive set, the compact-open transitive and point wise convergence topological transitive sets in $C(X, Y)$. Relationship between these new definitions is studied. Finally, we have introduced a number of very important topological concepts and shown that every compact-open convergence transitive set implies point wise transitive set and that every compact-open-mixing system implies point wise convergence system but not conversely. Finally, we have shown that every strongly compact-open-mixing set implies strongly point wise convergence mixing set but the converse not necessarily true.

\section{New Theorems of Point Wise-Convergence Topology}

Definition 2.1. Consider in $C(X, Y)$ the sets

$$
\left\{x_{i}, U_{i}\right\}_{i 1}^{k}=\left\{f \in C(X, Y): f\left(x_{i}\right) \in U_{i}, i=1, \cdots, k\right\}
$$

where $x_{1}, \cdots, x_{k} \in X, U_{1}, \cdots, U_{k}$ are open sets in $Y$.

The topology $\tau_{2}$ generated by these sets in their capacity as a subset is called the topology of point-wise convergence on $C(X, Y)$.

Note that any open set in $\tau_{2}$ is called pc-open set and $\left(C(X, Y), \tau_{2}\right)$ is called pc-topological space. The compliment of pc-open set is called pc-closed set.

Definition 2.2. A function $F: C(X, Y) \rightarrow C(X, Y)$ is called pc-irresolute if the inverse image of each pc-open set is a pc-open set in $C(X, Y)$.

Definition 2.3. A map $F: C(X, Y) \rightarrow C(X, Y)$ is $p c r$-homeomorphism if it is bijective and thus invertible and both $F$ and $F^{-1}$ are $p c$-irresolute.

The systems $F: C(X, X) \rightarrow C(X, X)$ and $G: C(Y, Y) \rightarrow C(Y, Y)$ are topologically pcr-conjugate if there is a pcr-homeomorphism $H: C(X, X) \rightarrow C(Y, Y)$ such that $H \circ F=G \circ H$.

Let $\left(C(X, Y), \tau_{2}\right)$ be a pc-topological space. The intersection of all pc-closed sets of $\left(C(X, Y), \tau_{2}\right)$ containing $A$ is called the pc-closure of $A$ and is denoted by $C l_{p c}(A)$.

Definition 2.4. Let $\left(C(X, Y), \tau_{2}\right)$ be a point wise convergence-topological space, and $F: C(X, Y) \rightarrow C(X, Y)$ be a map. The map $F$ is said to have pc-dense orbit if there exists $f \in C(X, Y)$ such that $C l_{p c}\left(O_{F}(f)\right)=C(X, Y)$.

Definition 2.5. Let $\left(C(X, Y), \tau_{2}\right)$ be a pc-topological space, and $F: C(X, Y) \rightarrow C(X, Y)$ be a pc-irresolute map, then $F$ is said to be a point-wise-converge-transitive (shortly pc-transitive) map if for every pair of pc-open sets $U$ and $V$ in $\left(C(X, Y), \tau_{2}\right)$ there is a positive integer $n$ such that $F^{n}(U) \cap V \neq \phi$.

Definition 2.6. Let $\left(C(X, Y), \tau_{2}\right)$ be a point wise convergence-topological space, and $F: C(X, Y) \rightarrow C(X, Y)$ be a pc-irresolute then the set $A \subseteq C(X, Y)$ is called pc-type transitive set if for every pair of non-empty pc-open sets $U$ and $V$ in $C(X, Y)$ with $A \cap U \neq \phi$ and $A \cap V \neq \phi$ there is a positive integer $\mathrm{n}$ such that $F^{n}(U) \cap V \neq \phi$. 
Definition 2.7. 1) Let $\left(C(X, Y), \tau_{1}\right)$ be a point-wise convergence-topological space, and $F: C(X, Y) \rightarrow C(X, Y)$ be a pc-irresolute then the set $A \subseteq C(X, Y)$ is called is called topologically pc-mixing set if, given any nonempty pc-open subsets $U, V \subseteq C(X, Y)$ with $A \cap U \neq \phi$ and $A \cap V \neq \phi$ then $\exists N>0$ such that $F^{n}(U) \cap V \neq \phi$ for all $n>N$.

2) The set $A \subseteq C(X, Y)$ is called a weakly pc-mixing set of $(C(X, Y), F)$ if for any choice of nonempty pc-open subsets $V_{1}, V_{2}$ of $A$ and nonempty pc-open subsets $U_{1}, U_{2}$ of $C(X, Y)$ with $A \cap U_{1} \neq \phi$ and $A \cap U_{2} \neq \phi$ there exists $n \in \mathrm{N}$ such that $F^{n}\left(V_{1}\right) \cap U_{1} \neq \phi$ and $F^{n}\left(V_{1}\right) \cap U_{2} \neq \phi$.

3) The set $A \subseteq C(X, Y)$ is strongly pc-mixing if for any pair of pc-open sets $U$ and $V$ with $U \cap A \neq \phi$ and $V \cap A \neq \phi$, there exist some $n \in \mathbf{N}$ such that $F^{k}(U) \cap V \neq \phi$ for any $k \geq n$.

4) Any element $f \in C(X, Y)$ such that its orbit $O_{F}(f)$ is pc-dense in $X$. is called hypercyclic element.

5) A system $(C(X, Y), F)$ is said to be topologically pc-mixing if, given pc-open sets $\mathrm{U}$ and $\mathrm{V}$ in $C(X, Y)$, there exists an integer $N$, such that, for all $n>N$, one has $F^{n}(U) \cap V \neq \phi$.

6) A system $(C(X, Y), F)$ is called topologically pc-mixing if for any non-emptypc-open set $\mathrm{U}$, there exists $n \in \mathrm{N}$ such that $\bigcup_{n \geq N} F^{n}(U)$ is pc-dense in $C(X, Y)$.

\section{Definitions and Theorems of Compact-Open Topology}

The following definition supplies another version of a topology on the set $C(X, Y)$.

Definition 3.1. Consider all possible sets of maps of the form [1]

$$
[K, U]=\{f \in C(X, Y): f(K) \subset U\}
$$

where $K$ is a compact set in $X$ and $U$ an open set in $Y$. The topology $\tau_{3}$ generated by these sets $[K, U]$ as a subbase is called the compact-open topology on $C(X, Y)$.

Note that any open set in $\tau_{3}$ is called co-open set and $\left(C(X, Y), \tau_{3}\right)$ is called co-topological space. The compliment of co-open set is called co-closed set.

Definition 3.2. Let $\left(C(X, Y), \tau_{3}\right)$ be a co-topological space. The map $F: C(X, Y) \rightarrow C(X, Y)$ is called co-irresolute if for every subset $A \in \tau_{3}$, $F^{-1}(A) \in \tau_{3}$. or, equivalently, $F$ is co-irresolute if and only if for every co-closed set $A, F^{-1}(A)$ is co-closed set.

Definition 3.3. A map $F: C(X, Y) \rightarrow C(X, Y)$ is cor-homeomorphism if it is bijective and thus invertible and both $F$ and $F^{-1}$ are co-irresolute.

The systems $F: C(X, X) \rightarrow C(X, X)$ and $G: C(Y, Y) \rightarrow C(Y, Y)$ are topologically cor-conjugate if there is a cor-homeomorphism $H: C(X, X) \rightarrow C(Y, Y)$ such that $H \circ F=G \circ H$.

Let $\left(C(X, Y), \tau_{3}\right)$ be a co-topological space. The intersection of all co-closed 
sets of $\left(C(X, Y), \tau_{3}\right)$ containing $\mathrm{A}$ is called the co-closure of $\mathrm{A}$ and is denoted by $C l_{c o}(A)$.

Definition 3.4. Let $\left(C(X, Y), \tau_{3}\right)$ be a compact-open topological space, and $F: C(X, Y) \rightarrow C(X, Y)$ be a map. The map $F$ is said to have co-dense orbit if there exists $f \in C(X, Y)$ such that $C l_{c o}\left(O_{F}(f)\right)=C(X, Y)$.

Definition 3.5. Let $\left(C(X, Y), \tau_{3}\right)$ be a co-topological space, and $F: C(X, Y) \rightarrow C(X, Y)$ be a co-irresolute map, then $F$ is said to be a compact-open-transitive ( shortly co-transitive) map if for every pair of co-open sets $U$ and $V$ in $\left(C(X, Y), \tau_{3}\right)$ there is a positive integer n such that $F^{n}(U) \cap V$ is not empty.

Definition 3.6. Let $\left(C(X, Y), \tau_{3}\right)$ be a co-topological space, and $F: C(X, Y) \rightarrow C(X, Y)$ be a co-irresolute then the set $A \subseteq C(X, Y)$ is called co-type transitive set if for every pair of non-empty co-open sets $U$ and $V$ in $C(X, Y)$ with $A \cap U \neq \phi$ and $A \cap V \neq \phi$ there is a positive integer $n$ such that $F^{n}(U) \cap V \neq \phi$.

Definition 3.7. 1) Let $\left(C(X, Y), \tau_{3}\right)$ be a co-topological space, and $F: C(X, Y) \rightarrow C(X, Y)$ be a co-irresolute then the set $A \subseteq C(X, Y)$ is called is called topologically co-mixing set if, given any nonempty co-open subsets $U, V \subseteq C(X, Y)$ with $A \cap U \neq \phi$ and $A \cap V \neq \phi$ then $\exists N>0$ such that $F^{n}(U) \cap V \neq \phi$ for all $n>N$.

2) The set $A \subseteq C(X, Y)$ is called a weakly co-mixing set of $(C(X, Y), F)$ if for any choice of nonempty co-open subsets $V_{1}, V_{2}$ of $A$ and nonempty co-open subsets $U_{1}, U_{2}$ of $C(X, Y)$ with $A \cap U_{1} \neq \phi$ and $A \cap U_{2} \neq \phi$ there exists $n \in \mathrm{N}$ such that $F^{n}\left(V_{1}\right) \cap U_{1} \neq \phi$ and $F^{n}\left(V_{1}\right) \cap U_{2} \neq \phi$.

3) The set $A \subseteq C(X, Y)$ is strongly co-mixing if for any pair of co-open sets $U$ and $V$ with $U \cap A \neq \phi$ and $V \cap A \neq \phi$, there exist some $n \in \mathbf{N}$ such that $F^{k}(U) \cap V \neq \phi$ for any $k \geq n$.

4) A system $(C(X, Y), F)$ is said to be topologically co-mixing if, given co-open sets $\mathrm{U}$ and $\mathrm{V}$ in $C(X, Y)$, there exists an integer $N$, such that, for all $n>N$, one has $F^{n}(U) \cap V \neq \phi$. For related works about weakly mixing see [2], [3] and [4].

\section{Conclusions}

We have the following results:

1) Every compact-open-transitive set implies point wise convergence set but not conversely.

2) Every compact-open-mixing system implies point wise convergence system but not conversely.

3) Every strongly compact-open-mixing set implies strongly point wise convergence mixing set.

\section{Acknowledgements}

First, thanks to my family for having the patience with me for having taking yet 
another challenge which decreases the amount of time I can spend with them. Specially, my wife who has taken a big part of that sacrifices, also, my son Sarmad who helps me for typing my research. Thanks to all my colleagues for helping me for completing my research.

\section{Conflicts of Interest}

The author declares no conflicts of interest regarding the publication of this paper.

\section{References}

[1] Borsovich, Y.U., Blizntakov, N., Izrailevich, Y.A. and Fomenko, T. (1985) Introduction to Topology. Mir Publisher, Moscow.

[2] Chacon, R.V. (1969) Weakly Mixing Transformations Which Are Not Strongly Mixing. Proceedings of the American Mathematical Society, 22, 559-562. https://doi.org/10.1090/S0002-9939-1969-0247028-5

[3] Kaki, M.N.M. (2015) Chaos: Exact, Mixing and Weakly Mixing Maps. Pure and Applied Mathematics Journal, 4, 39-42. https://doi.org/10.11648/j.pamj.20150402.11

[4] Kaki, M.N.M. (2013) Introduction to Weakly b-Transitive Maps on Topological Space. Science Research, 1, 59-62. https://doi.org/10.11648/j.sr.20130104.11 